\title{
Condensate fraction in neutron matter
}

\author{
Luca Salasnich \\ Dipartimento di Fisica "Galileo Galilei" and CNISM, \\ Università di Padova, Via Marzolo 8, 35131 Padova, Italy
}

(Dated: November 11, 2018)

\begin{abstract}
We study the Bose-Einstein condensation of fermionic pairs in the uniform neutron matter by using the concept of the off-diagonal long-range order of the two-body density matrix of the system. We derive explicit formulas for the condensate density $\rho_{\text {con }}$ and the condensate fraction $\rho_{\text {con }} / \rho$ in terms of the scaled pairing energy gap $\Delta / \epsilon_{F}$ where $\epsilon_{F}$ is the Fermi energy. We calculate the condensate fraction $\rho_{\text {con }} / \rho$ as a function of the density $\rho$ by using previously obtained results for the pairing gap $\Delta$. We find the maximum condensate fraction $\left(\rho_{\text {con }} / \rho\right)_{\max }=0.42$ at the density $\rho=5.3 \cdot 10^{-4} \mathrm{fm}^{-3}$, which corresponds to the Fermi wave number $k_{F}=0.25 \mathrm{fm}^{-1}$.

PACS numbers: 21.65.Cd, 03.75.Ss
\end{abstract}

The concept of off-diagonal long-range order (ODLRO) was introduced by Penrose and Onsager [1] to determine the condensate fraction of a generic bosonic many-body system. Yang 2] extended this powerful idea to the case of fermionic many-body systems. It is now established that at zero temperature the condensate fraction of liquid ${ }^{4} \mathrm{He}$ is below $10 \%$ [3], while for dilute and ultracold bosonic alkali-metal atoms it can reach $100 \%$ [4]. Few years ago the condensate fraction has been calculated for fermionic atoms [5, 6] in the crossover from the BardeenCooper-Schrieffer (BCS) state of Cooper Fermi pairs to the Bose-Einstein condensate (BEC) of molecular dimers at zero temperature. These theoretical predictions are in quite good agreement with the data obtained in two experiments [7, 8] with ultra-cold two hyperfine component Fermi vapors of ${ }^{6} \mathrm{Li}$ atoms. Very recently, the condensate fraction of dilute neutron matter, which is predicted to fill the crust of neutron stars 9], has been calculated by Wlazlowski and Magierski [10, 11]. Unfortunately, these finite-temperature Path Integral Monte Carlo simulations are performed only at $\rho=0.003 \mathrm{fm}^{-3}$, and their trend suggests a condensate fraction of about $35 \%$ at zero temperature [11].

In this paper we present a zero-temperature systematic study of the condensate fraction of neutron matter as a function of the density $\rho$. First we derive explicit analytical formulas for the condensate density $\rho_{c o n}$ and the condensate fraction $\rho_{\text {con }} / \rho$ as a function of the pairing energy gap $\Delta$ in units of the Fermi energy $\epsilon_{F}$. With the help of these expressions we calculate the condensate fraction $\rho_{\text {con }} / \rho$ vs $\rho$ by using previously obtained results for the paring gap $\Delta[12,13]$.

The shifted Hamiltonian of the uniform neutron matter is given by

$$
\begin{aligned}
\hat{H}^{\prime} & =\int d^{3} \mathbf{r} \sum_{\sigma=\uparrow, \downarrow} \hat{\psi}_{\sigma}^{+}(\mathbf{r})\left(-\frac{\hbar^{2}}{2 m} \nabla^{2}-\mu\right) \hat{\psi}_{\sigma}(\mathbf{r}) \\
& +\int d^{3} \mathbf{r} d^{3} \mathbf{r}^{\prime} \hat{\psi}_{\uparrow}^{+}(\mathbf{r}) \hat{\psi}_{\downarrow}^{+}\left(\mathbf{r}^{\prime}\right) V\left(\mathbf{r}-\mathbf{r}^{\prime}\right) \hat{\psi}_{\downarrow}\left(\mathbf{r}^{\prime}\right) \hat{\psi}_{\uparrow}(\mathbf{r}),
\end{aligned}
$$

where $\hat{\psi}_{\sigma}(\mathbf{r})$ is the field operator that annihilates a neutron of spin $\sigma$ in the position $\mathbf{r}$, while $\hat{\psi}_{\sigma}^{+}(\mathbf{r})$ creates a neutron of spin $\sigma$ in $\mathbf{r}$. Here $V\left(\mathbf{r}-\mathbf{r}^{\prime}\right)$ is the nucleonnucleon potential in the ${ }^{1} \mathrm{~S}_{0}$ channel. The ground-state average of the number of neutrons reads

$$
N=\int d^{3} \mathbf{r} \sum_{\sigma=\uparrow, \downarrow}\left\langle\hat{\psi}_{\sigma}^{+}(\mathbf{r}) \hat{\psi}_{\sigma}(\mathbf{r})\right\rangle=\int d^{3} \mathbf{r}\langle\hat{\rho}(\mathbf{r})\rangle
$$

This total number $N$ is fixed by the chemical potential $\mu$ which appears in Eq. (11).

As shown by Yang [2], for a Fermi system ODLRO means that the two-body density matrix factorizes as follows

$$
\begin{array}{r}
\left\langle\hat{\psi}_{\uparrow}^{+}\left(\mathbf{r}_{1}^{\prime}\right) \hat{\psi}_{\downarrow}^{+}\left(\mathbf{r}_{2}^{\prime}\right) \hat{\psi}_{\downarrow}\left(\mathbf{r}_{1}\right) \hat{\psi}_{\uparrow}\left(\mathbf{r}_{2}\right)\right\rangle \\
=\left\langle\hat{\psi}_{\uparrow}^{+}\left(\mathbf{r}_{1}^{\prime}\right) \hat{\psi}_{\downarrow}^{+}\left(\mathbf{r}_{2}^{\prime}\right)\right\rangle\left\langle\hat{\psi}_{\downarrow}\left(\mathbf{r}_{1}\right) \hat{\psi}_{\uparrow}\left(\mathbf{r}_{2}\right)\right\rangle
\end{array}
$$

in the limit wherein both unprimed coordinates approach an infinite distance from the primed coordinates. The largest eigenvalue $N_{0}$ of the two-body density matrix gives the number of neutron pairs which have their center of mass with zero linear momentum. This condensed number of pairs is given by $[\underline{5}, 6]$

$$
N_{c o n}=2 \int d^{3} \mathbf{r}_{1} d^{3} \mathbf{r}_{2}\left|\left\langle\hat{\psi}_{\downarrow}\left(\mathbf{r}_{1}\right) \hat{\psi}_{\uparrow}\left(\mathbf{r}_{2}\right)\right\rangle\right|^{2} .
$$

Within the Hartree-Fock-Bogoliubov approach [14], the shifted Hamiltonian density (11) can be diagonalized by using the Bogoliubov-Valatin representation of the field operator $\hat{\psi}_{\sigma}(\mathbf{r})$ in terms of the anticommuting quasiparticle Bogoliubov operators $\hat{b}_{\mathbf{k} \sigma}$ with amplitudes $u_{\mathbf{k}}$ and $v_{\mathbf{k}}$ and quasi-particle energy $E_{\mathbf{k}}$. After minimization of the resulting quadratic Hamiltonian one finds familiar expressions for these quantities [14]:

$$
E_{\mathbf{k}}=\left[\left(\tilde{\epsilon}_{\mathbf{k}}-\mu\right)^{2}+\Delta_{\mathbf{k}}^{2}\right]^{1 / 2}
$$

and

$$
u_{\mathbf{k}}^{2}=\frac{1}{2}\left(1+\frac{\tilde{\epsilon}_{\mathbf{k}}-\mu}{E_{\mathbf{k}}}\right), \quad v_{\mathbf{k}}^{2}=\frac{1}{2}\left(1-\frac{\tilde{\epsilon}_{\mathbf{k}}-\mu}{E_{\mathbf{k}}}\right),
$$




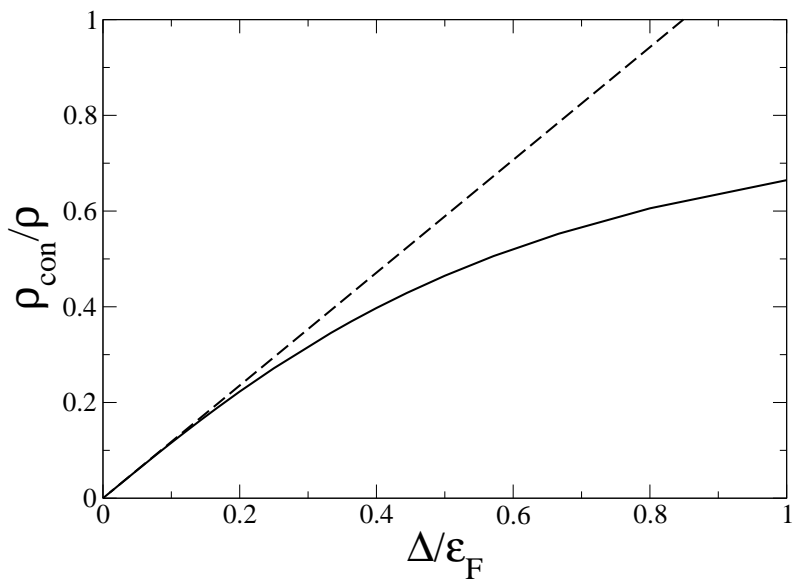

FIG. 1: Condensate fraction $\rho_{\text {con }} / \rho$ as a function of the scaled pairing gap $\Delta / \epsilon_{F}$. The solid line is obtained by using Eq. (14). The dashed line is obtained by using Eq. (15), valid in the regime $\Delta / \epsilon_{F} \ll 1$.

where $\tilde{\epsilon}_{\mathbf{k}}$ is the single-particle energy and $\Delta_{\mathbf{k}}$ is the pairing gap, which satisfies the integral equation

$$
\Delta_{\mathbf{q}}=\frac{1}{2} \sum_{\mathbf{k}}\langle\mathbf{q},-\mathbf{q}|V| \mathbf{k},-\mathbf{k}\rangle \frac{\Delta_{\mathbf{k}}}{E_{\mathbf{k}}}
$$

In the Hartree-Fock-Bogoliubov approach [14] the singleparticle energy $\tilde{\epsilon}_{k}$ is given by $\tilde{\epsilon}_{\mathbf{k}}=\epsilon_{\mathbf{k}}+U_{\mathbf{k}}$, where $\epsilon_{\mathbf{k}}=\hbar^{2} k^{2} /(2 m)$ is the bare single-particle energy and $U_{\mathbf{k}}=\sum_{\mathbf{q}}\langle\mathbf{k}, \mathbf{q}|V| \mathbf{k}, \mathbf{q}\rangle$ is the Hartree-Fock potential. The equation for the average number of neutrons is easily obtained from Eq. (2) as

$$
N=2 \sum_{\mathbf{k}} v_{\mathbf{k}}^{2}
$$

Finally, from Eq. (4) one finds that the condensate number of paired neutrons is given by [5, 6]

$$
N_{c o n}=2 \sum_{\mathbf{k}} u_{\mathbf{k}}^{2} v_{\mathbf{k}}^{2}
$$

The numerical solution of Eqs. (17), (8), and (9) is a hard computational task. However, the problem can be simplified under some reliable assumptions [14]. In Eqs. (6) we adopt the approximations $\tilde{\epsilon}_{\mathbf{k}} \simeq \epsilon_{\mathbf{k}}+U_{k_{F}}$ and $\mu \simeq \epsilon_{k_{F}}+U_{k_{F}}$, where $k_{F}=\left(3 \pi^{2} \rho\right)^{1 / 3}$ is the Fermi wavenumber of the non-interacting Fermi gas with density $\rho$. In this way we get $\tilde{\epsilon}_{\mathbf{k}}-\mu \simeq \hbar^{2} k^{2} /(2 m)-\epsilon_{F}$ where $\epsilon_{F}=\epsilon_{k_{F}}=\hbar^{2}\left(3 \pi^{2} \rho\right)^{2 / 3} /(2 m)$ is the Fermi energy of the non-interacting Fermi gas. Notice that this is formally equivalent of setting directly $\tilde{\epsilon}_{\mathbf{k}} \simeq \epsilon_{\mathbf{k}}$ and $\mu \simeq \epsilon_{F}$ in Eqs. (6). In addition, we set $\Delta_{\mathrm{k}} \simeq \Delta_{k_{F}}=\Delta$. Under these assumptions, in the three-dimensional continuum limit the gap equation (17) of the neutron matter becomes

$$
1=\frac{1}{2} \int \frac{d^{3} \mathbf{k} d^{3} \mathbf{r}}{(2 \pi)^{3}} \frac{V(\mathbf{r}) e^{i\left(\mathbf{k}-\mathbf{k}_{F}\right) \cdot \mathbf{r}}}{\sqrt{\left(\frac{\hbar^{2} k^{2}}{2 m}-\epsilon_{F}\right)+\Delta^{2}}} .
$$

Moreover, from the number equation (8) we find the total density as

$$
\rho=\frac{1}{2} \frac{(2 m)^{3 / 2}}{2 \pi^{2} \hbar^{3}} \Delta^{3 / 2} I\left(\frac{\epsilon_{F}}{\Delta}\right)
$$

where $I(x)$ is the monotonic function

$$
I(x)=\int_{0}^{+\infty} y^{2}\left(1-\frac{y^{2}-x}{\sqrt{\left(y^{2}-x\right)^{2}+1}}\right) d y .
$$

In a similar way from Eq. (9) we get the condensate density of the neutron-neutron pair

$$
\rho_{c o n}=\frac{m^{3 / 2}}{8 \pi \hbar^{3}} \Delta^{3 / 2} \sqrt{\frac{\epsilon_{F}}{\Delta}+\sqrt{1+\frac{\epsilon_{F}^{2}}{\Delta^{2}}}} .
$$

The condensate fraction follows immediately as the ratio between $\rho_{\text {con }}$, given by Eq. (13) and $\rho$, given by Eq. (11), namely

$$
\frac{\rho_{c o n}}{\rho}=\frac{\pi}{2^{5 / 2}} \frac{\sqrt{\frac{\epsilon_{F}}{\Delta}+\sqrt{1+\frac{\epsilon_{F}^{2}}{\Delta^{2}}}}}{I\left(\frac{\epsilon_{F}}{\Delta}\right)}
$$

Notice that this fraction is expressed only in terms of the ratio $\epsilon_{F} / \Delta$. In the deep BCS regime where $\Delta / \epsilon_{F} \ll$ 1 then $\sqrt{\epsilon_{F} / \Delta+\sqrt{1+\epsilon_{F}^{2} / \Delta^{2}}} \simeq \sqrt{2}\left(\epsilon_{F} / \Delta\right)^{1 / 2}$ and $I\left(\epsilon_{F} / \Delta\right) \simeq(2 / 3)\left(\epsilon_{F} / \Delta\right)^{3 / 2}$ and consequently the condensate fraction becomes

$$
\frac{\rho_{\text {con }}}{\rho}=\frac{3 \pi}{8} \frac{\Delta}{\epsilon_{F}} .
$$

In Fig. 1 we plot the condensate fraction $\rho_{\text {con }} / \rho$ as a function of the scaled pairing gap $\Delta / \epsilon_{F}$. The solid line is obtained by using Eq. (14), while the dashed line is obtained by using Eq. (15). The figure shows that for $\Delta / \epsilon_{F} \leq 0.2$ the two curves practically coincide.

To estimate the condensate fraction $\rho_{c o n} / \rho$ in neutron matter on the basis of Eq. (14) it is necessary to obtain $\Delta / \epsilon_{F}$ from Eq. (10) or, if possible, directly from Eq. (7). This problem has been investigated by various authors 12, 13, 15 21. For very dilute neutron matter, the nucleon-nucleon potential $V\left(\mathbf{r}-\mathbf{r}^{\prime}\right)$ in the ${ }^{1} S_{0}$ channel can be parametrized in terms of only the s-wave scattering length $a_{s}=-18.5 \mathrm{fm}$ [14]. Indeed, in the regime $k_{F}\left|a_{s}\right| \ll 1$, the pairing gap is exponentially small and from Eq. (10) one finds [14]

$$
\frac{\Delta}{\epsilon_{F}}=\frac{8}{e^{2}} e^{-\pi /\left(2 k_{F}\left|a_{s}\right|\right)} .
$$

It follows immediately from Eq. (15) that in this regime of very-low density (BCS regime) the condensate fraction reads

$$
\frac{\rho_{c o n}}{\rho}=\frac{3 \pi}{e^{2}} e^{-\pi /\left(2 k_{F}\left|a_{s}\right|\right)} .
$$




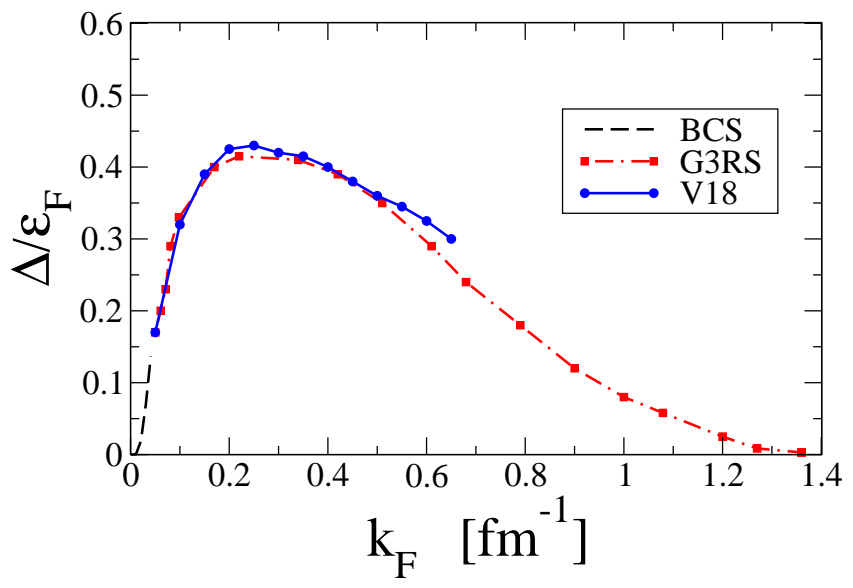

FIG. 2: (Color online). Scaled pairing gap $\Delta / \epsilon_{F}$ of neutron matter as a function of the Fermi wave number $k_{F}$. The dashed line is obtained with Eq. (16); the filled squares with the dot-dashed line are the results obtained with the $G 3 R S$ nuclear potential [12]; the filled circles with the solid line are the results obtained with the Argone $V 18$ nuclear potential [13].

At higher densities the pairing gap $\Delta$ does not follow an exponential grow but instead it reaches a maximum and eventually goes to zero [12, 13, 15 21]. In Fig. 2 we report the scaled pairing gap $\Delta / \epsilon_{F}$ as a function of the Fermi wave number $k_{F}$. The solid line, obtained with Eq. (16), shows the exponential grow which is reliable only in the very low density BCS regime. The filled squares are the results obtained by Matsuo [12] by solving Eq. (7) with the G3RS nuclear potential [22]; the filled circles are the results obtained by Gezerliz and Carlson [13] with the Argone V18 nuclear potential [23]. The figure shows that the two nucleon-nucleon potentials give very similar results.

Instead of solving Eq. (10) we use the data of $\Delta / \epsilon_{F}$ shown in Fig. 2 to derive the condensate fraction $\rho_{\text {con }} / \rho$ by using our Eq. (14). It is then physically relevant to plot the obtained condensate fraction $\rho_{\text {con }} / \rho$ as a function of the density $\rho=k_{F}^{3} /\left(3 \pi^{2}\right)$.

The results are shown in Fig. 3, where the horizontal axis is in logarithmic scale. At very low density $\rho$ the neutron matter behaves like a quasi-ideal Fermi gas with weakly correlated Cooper pairs and the condensate fraction $\rho_{c o n} / \rho$ is exponentially small (BCS regime, dashed line). By increasing the density $\rho$ the attractive tail of the neutron-neutron potential becomes relevant and the condensate fraction $\rho_{\text {con }} / \rho$ grows significantly. On the basis of the data obtained with the Argone $V 18$ potential (filled circles with dot-dashed line), the maximum of the condensate fraction is $\left(\rho_{\text {con }} / \rho\right)_{\max }=0.42$ at the density $\rho=5.3 \cdot 10^{-4} \mathrm{fm}^{-3}$ which corresponds to the Fermi wave number $k_{F}=0.25 \mathrm{fm}^{-1}$. By further increasing the density $\rho$ the repulsive core of the neutron-neutron potential plays an important role in destroying the correlation of Cooper pairs and the condensate fraction $\rho_{\text {con }} / \rho$ slowly

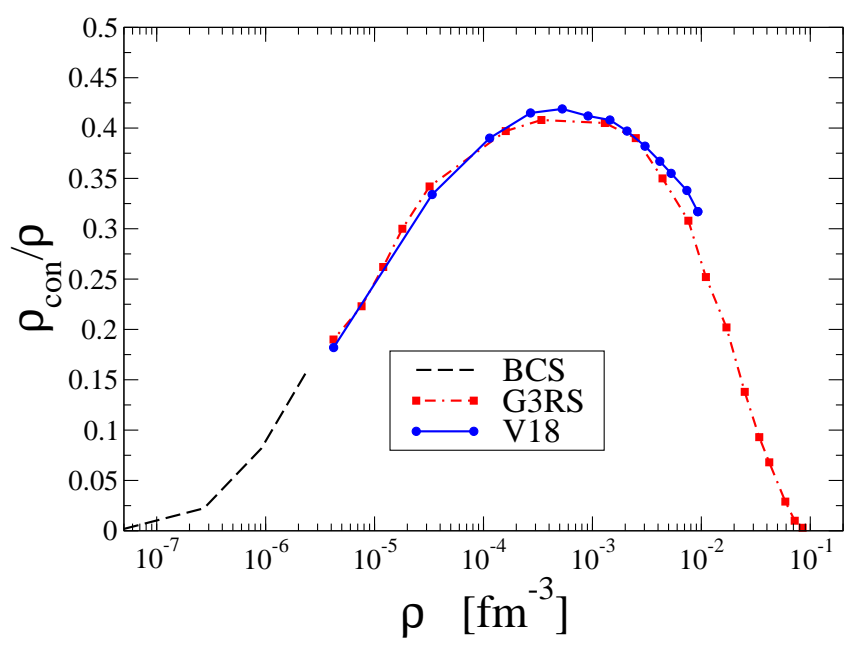

FIG. 3: (Color online). Condensate fraction $\rho_{\text {con }} / \rho$ of neutron matter as a function of the density $\rho$. It is derived by using the data of $\Delta / \epsilon_{F}$ of Fig. 2 and Eq. (14).

goes to zero (filled squares with solid line). Remarkably, the results of Fig. 1 are fully consistent with the Monte Carlo value $\rho_{\text {con }} / \rho \simeq 0.35$ at $\rho=0.003 \mathrm{fm}^{-3}$ one extracts from the finite-temperature Path Integral Monte Carlo data of Wlazlowski and Magierski [10, 11].

As discussed in the introduction, the properties of lowdensity neutron matter are important for the understanding of neutron star crusts [9] and also the exterior of neutron-rich nuclei 24]. Our present investigation gives a contribution to this fascinating field of research: the condensate fraction of neutron matter reaches its maximum value of about $40 \%$ in correspondence to the maximum of the pairing gap at the density of about $5 \cdot 10^{-4}$ $\mathrm{fm}^{-3}$, which is much smaller than the nuclear saturation density $0.16 \mathrm{fm}^{-3}$. It is important to stress that neutron-neutron pairs do not form a true bound state and the system cannot reach the unitarity limit (where there is the formation of the bound state) nor the deep BEC regime characterized by a gas of di-neutrons with $100 \%$ condensate fraction [12]. Nevertheless, the maximum value of the condensate fraction we have found for neutron matter is not too far from to the value of about $70 \%$ calculated for the unitarity Fermi gas [5]. Indeed our results clearly show a BCS-quasiunitary-BCS crossover by increasing the neutron density.

The author thanks Prof. Masayuki Matsuo for making available his numerical data and for useful suggestions. 
[3] D.M. Ceperly, Rev. Mod. Phys. 67, 2 (1995).

[4] C. Pethick and H. Smith, Bose-Einstein Condensation in Dilute Gases (Cambridge University Press, Cambridge, 2002).

[5] L. Salasnich, N. Manini, and A. Parola, Phys. Rev. A 72, 023621 (2005).

[6] G. Ortiz and J. Dukelsky, Phys. Rev. A 72, 043611 (2005).

[7] M.W. Zwierlein, C.H. Schunck, C.A. Stan, S.M.F. Raupach, and W. Ketterle, Phys. Rev. Lett. 94, 180401 (2005).

[8] Y. Inada, M. Horikoshi, S. Nakajima, M. KuwataGonokami, M. Ueda, and T. Mukaiyama, Phys. Rev. Lett. 101, 180406 (2008).

[9] C.J. Pethick and D.G. Ravenhall, Ann. Rev. Nucl. Part. Sci. 45, 429 (1995).

[10] G. Wlazlowski and P. Magierski, Phys. Rev. C 83, 012801(R) (2011).

[11] G. Wlazlowski and P. Magierski, Int. J. Mod. Phys. E 20, 569 (2011).

[12] M. Matsuo, Phys. Rev. C 73, 044309 (2006).

[13] A. Gezerlis and J. Carlson, Phys. Rev. C 81, 025803 (2010).
[14] A. Fetter and J.D. Walecka, Quantum Theory of ManyParticle Systems (Dover, New York, 2003).

[15] T. Alm, B.L. Friman, G. Ropke, and H. Shulz, Nucl. Phys. A 551, 45 (1993)

[16] G. Lazzari and F.V. De Blasio, Act. Phys. Pol. B 25, 1467 (1994).

[17] M. Baldo, U. Lombardo, and P. Schuck, Phys. Rev. C 52, 975 (1995).

[18] O Elgaroy and M. Hjorth-Jensen, Phys. Rev. C 57, 1174 (1998).

[19] A. Sedrakian and J.W. Clark, Phys. Rev. 73, 035803 (2006).

[20] J. Margueron, H. Sagawa, and K. Hagino, Phys. Rev. 76, 064316 (2007).

[21] S. Mao, X. Huang, and P. Zhuang, Phys. Rev. C 79, 034304 (2009).

[22] R. Tamagaki, Progr. Theor. Phys. 89, 91 (1967).

[23] R.B. Wiringa, V.G.J. Stoks, and R. Schiavilla, Phys. Rev. C 51, 38 (1995).

[24] N. Chamel, S. Goriely, and J.M. Pearson, Nucl. Phys. A 812, 72 (2008). 